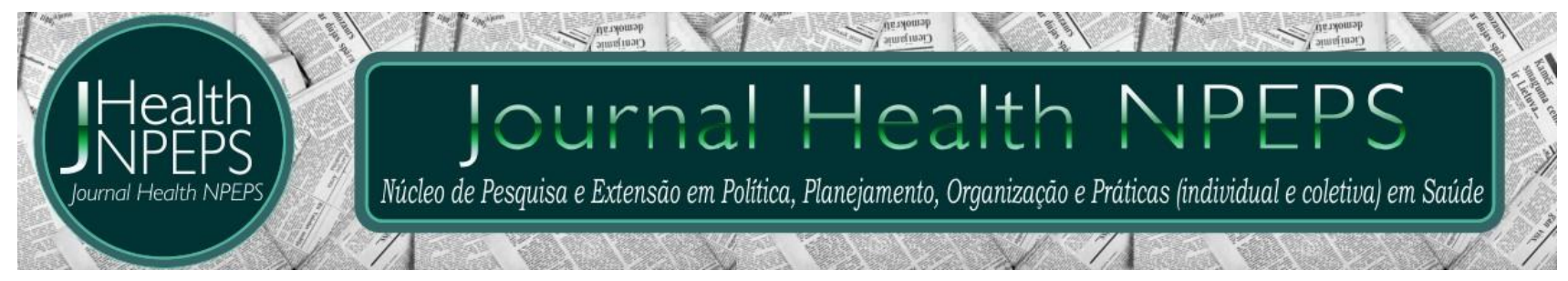

http://dx.doi.org/10.30681/252610104630

ARTIGO ORIGINAL

\title{
Avaliação da qualidade de vida e perfil socioeconômico em diabéticos insulinodependentes
}

\section{Assessment of the quality of life and socioeconomic profile of insulin-dependent diabetics}

\section{Avaliação da qualidade de vida e perfil socioeconómico em diabéticos insulinodependentes}

\section{Gabriele Barros de Aviz¹, Flávia Marques Santos², Valéria Diniz Calandrini de Azevedo $^{3}$, Giovanna Gomes e Silva ${ }^{4}$, Lídia Lacerda Furtado ${ }^{5}$}

\section{RESUMO}

Objetivo: avaliar como a insulinoterapia reflete no cotidiano dos portadores de diabetes mellitus. Método: os dados foram coletados com uma amostra total de 70 pacientes com diagnóstico prévio de diabetes mellitus em uso de insulina. Foram aplicados questionários sobre perfil socioeconômico e qualidade de vida por meio da versão brasileira da escala PAID. Após a coleta, o teste D’Agostino-Pearson foi utilizado para a estatística, sendo executado com o auxílio do programa BioEstat 5.5. Resultados: predominou pacientes do sexo feminino (64,3\%), com ensino fundamental $(44,3 \%)$ e renda de até um salário mínimo (75,7\%). O tempo médio de diagnóstico foi de 12,4 anos, com uso de insulina por pelo menos cinco anos. Observou-se que $64,3 \%$ dos pacientes apresentavam escore B-PAID elevado $(\geq 40)$, o que indica alto nível de sofrimento emocional relacionado ao diabetes. Conclusão: identificou um impacto negativo na qualidade de vida dos participantes do estudo, apontando necessidade de melhor suporte clínico e emocional.

Descritores: Diabetes Mellitus; Insulina; Qualidade de Vida.

\section{ABSTRACT}

\footnotetext{
${ }^{1}$ Médica. Centro Universitário do Estado do Pará. Belém, Pará, Brasil. E-mail: gabrielebaviz@hotmail.com ORCID ID: http://orcid.org/0000-0002-7009-9080 Autor para correspondência - Endereço: Avenida Almirante Barroso, 3775, CEP 66612903. Belém, Pará, Brasil.

2Médica. Universidade Federal do Pará. Belém, Pará, Brasil. E-mail: drafms@gmail.com ORCID ID: http://orcid.org/0000-0002-0923$\underline{4191}$

${ }^{3}$ Médica. Centro Universitário do Estado do Pará. Belém, Pará, Brasil. E-mail: valedinizcalandrini@gmail.com ORCID ID: http://orcid.org/0000-0002-5701-197X

${ }^{4}$ Médica. Centro Universitário do Estado do Pará. Belém, Pará, Brasil. E-mail: gigomes1@hotmail.com ORCID ID: http://orcid.org/0000-0002-4826-2785

${ }_{5}^{5}$ Médica. Centro Universitário do Estado do Pará. Belém, Pará, Brasil. E-mail: lacerdafurtadolidia@gmail.com ORCID ID: http://orcid.org/0000-0003-4982-1232
} meio, desde que a publicação original seja corretamente citada. 
Objective: to evaluate how insulin therapy reflects in the daily lives of people with diabetes mellitus. Method: the data were collected with a total sample of 70 patients with a previous diagnosis of diabetes mellitus using insulin. Questionnaires on socioeconomic profile and quality of life were applied using the Brazilian version of the PAID scale. After collection, the D'Agostino-Pearson test was used for statistics, being performed with the aid of the BioEstat 5.5 program. Results: the protocol used observed that the majority of patients were female (64.3\%), attended elementary school (44.3\%) and had an income of up to 1 minimum wage (75.7\%). The average time of diagnosis was 12.4 years, using insulin for at least 5 years. It was observed that the majority (64.3\%) of the patients had a high B-PAID score $(\geq 40)$, which indicates a high level of emotional distress related to diabetes. Conclusion: a negative impact on the quality of life of the study participants, pointing out the need for better clinical and emotional support was identified.

Descriptors: Diabetes Mellitus; Insulin; Quality of Life.

\section{RESUMEN}

Objetivo: evaluar cómo se refleja la terapia con insulina en la vida diaria de las personas con diabetes mellitus. Método: los datos se recogieron con una muestra total de 70 pacientes con diagnóstico previo de diabetes mellitus utilizando insulina. Se aplicaron cuestionarios sobre perfil socioeconómico y calidad de vida utilizando la versión brasileña de la escala PAID. Después de la recolección, se utilizó la prueba D’Agostino-Pearson para estadísticas, que se realizó con la ayuda del programa BioEstat 5.5. Resultados: el protocolo utilizado observó que la mayoría de los pacientes eran mujeres (64.3\%), asistían a la escuela primaria (44.3\%) y tenían un ingreso de hasta 1 salario mínimo (75.7\%). El tiempo promedio de diagnóstico fue de 12.4 años, usando insulina durante al menos 5 años. Se observó que la mayoría (64,3\%) de los pacientes tenían una puntuación alta de B-PAID ( $\geq 40)$, lo que indica un alto nivel de angustia emocional relacionada con la diabetes. Conclusion: identificó un impacto negativo en la calidad de vida de los participantes del estudio, señalando la necesidad de un mejor apoyo clínico y emocional.

Descriptores: Diabetes Mellitus; Insulina; Calidad de Vida.

\section{INTRODUÇÃO}

Diabetes Mellitus (DM) é uma

doença metabólica crônica,

caracterizada por hiperglicemia, sendo causada por fatores genéticos e ambientais $^{1}$. Essa doença configura uma epidemia mundial. Atualmente, a Organização Mundial de Saúde (OMS) estima que 246 milhões de pessoas possuam diabetes. A estimativa atual no
Brasil é de que 11,9 milhões de habitantes entre 20 a 79 anos sejam diabéticos, o que equivale a quase $6 \% \mathrm{da}$ população brasileira².

Diversos fatores de risco para esse desfecho estão apontados na literatura, como sedentarismo, estilo de vida pouco saudável e obesidade, os quais vem sendo os grandes responsáveis pelo aumento da incidência da doença ${ }^{3}$.

Para o devido tratamento da 
doença pode lançar mão da insulina, a qual é essencial no tratamento de Diabetes Mellitus tipo 1 (DM1), enquanto que nos pacientes com Diabetes Mellitus tipo 2 (DM2) é frequentemente protelada até condições de grave descompensação metabólica ou na presença de complicações que não seja possível manter medicações por via oral ${ }^{4}$. Em população de baixa renda há maior risco de controle metabólico glicêmico desfavorável podendo antecipar a insulinoterapia 5 .

Seu uso, além de controlar o nível glicêmico, diminui as complicações, doenças cardiovasculares, insuficiência renal, cegueira e amputação de membros ${ }^{6}$. 0 que muitas vezes limita o alcance do controle glicêmico é a falta de adesão ao tratamento, suporte familiar inadequado, agravos gerados pelo efeito da insulina e/ou pela má administração durante o tratamento 7 .

O impacto do uso de insulina na Qualidade de Vida (QV) de seus usuários é contraditório ${ }^{8}$. Apesar de seus benefícios, possui diversas limitações no controle glicêmico como injeções dolorosas, múltiplas aplicações, impedimentos no dia a dia e até efeitos adversos como lipoadistrofias e infecções locais. Tais problemas contribuem para insatisfação com tratamento e geram ansiedade, desestímulos e outros fatores emocionais que interferem de maneira negativa a QV e, consequentemente, no controle da doença?.

A QV é definida pela OMS como a percepção do indivíduo sobre sua posição na vida no contexto da cultura, sistema de valores nos quais ela vive e em relação aos objetivos, expectativas, padrões de atitude e preocupações ${ }^{13}$. Para isso, a literatura dispõe de vários instrumentos para avaliar essa condição em populações sadias ou não ${ }^{14}$.

Pesquisas revelam que a $\mathrm{QV}$ em pacientes com DM é menor do que aqueles sem a doença e algumas variáveis como idade, complicações, nível social, fatores psicológicos, educação, tipo de assistência, tempo de diagnóstico, entre outras, podem interferir ${ }^{10}$.

Em estudo sobre a comparação da insulinoterapia com outras modalidades terapêuticas para diabetes, evidenciou que a terapia com insulina pode gerar níveis maiores de estresse emocional ${ }^{10,11}$. Esse quadro psicoemocional é composto por frustações e descrença no caráter crônico da doença; sobrecarga ou desanimação com seu manejo, medo, depressão e ansiedade, as quais afetam 
a aderência ao tratamento ${ }^{17,18}$.

Dessa forma, o estudo teve o objetivo de avaliar como a insulinoterapia reflete no cotidiano dos portadores de DM. Tal desfecho, no cotidiano, tem 0 potencial de demonstrar o perfil desses pacientes, 0 estado de saúde e o bem-estar deste grupo a fim de subsidiar estratégias de intervenções mais eficientes que visem melhorar a QV da população ${ }^{16}$.

\section{MÉTODO}

O estudo é do tipo descritivo e transversal. Foi utilizado a declaração Strengthening the Reporting of Observational studies in Epidemiology (STROBE), diretriz para relatar estudos observacionais. Foi realizado nos ambulatórios de metabologia e DM1 do Centro de Especialidades Médicas do CESUPA (CEMEC). A amostra foi composta por 70 pacientes, usuários Sistema Único de Saúde (SUS) diagnosticados com DM1 e DM2 em uso de insulina de ambos os sexos com 18 anos ou mais que foram atendidos entre fevereiro e julho de 2019. Foram excluídos gestantes e portadores de doenças crônicas graves, a partir do acesso nos prontuários médicos.
Para a coleta de dados foram aplicados dois questionários. 0 primeiro levantou dados clínico (tempo de diagnóstico, tempo de insulinoterapia e frequência de aplicações diárias) e sociodemográficos (idade, escolaridade e gênero) por meio de um questionário do criado pelo autor e aplicado sob forma de entrevista. O segundo sobre QV foram coletadas por meio da versão brasileira da escala Problem Areas in Diabetes (PAID), questionário de 20 itens, focado nos aspectos emocionais negativos em relação à doença. 0 questionário foi criado por Polonsky e colaboradores em 1995 para verificar a QV dos pacientes com diagnósticos de DM e foi traduzido por meio de um processo retradução e tradução conforme recomendado por Clare Bradley, sendo comparado aos modelos já existentes de avaliação de QV como a Escala Resumida de Qualidade de Vida da OMS e a Escala de Satisfação de Vida com coeficiente Alpha de Crombach de $0,93^{15}$. As questões encontram-se divididas em quatro áreas específicas: 12 questões referentes a problemas emocionais relacionados ao viver com diabetes, três referentes a problemas relacionados com 0 tratamento, três referentes a problemas relacionados com à alimentação, e duas 
referentes a problemas relacionados ao apoio social.

O questionário já estruturado na forma de escala Likert, que variam de 0 (não é um problema), a 4 (é um problema sério). Ao final do questionário, produz um escore total que varia de 0 a 100. Ao multiplicar a pontuação gerada pelas 20 perguntas por 1,25 , se o escore BPAID for $\geq 40$, indica alto nível de sofrimento emocional relacionado ao diabetes.

As informações obtidas foram colocadas em planilha no Microsoft Excel 2007. Para análise de dados as variáveis foram expressas sob a forma de média, desvio padrão, mediana e desvio interquartílico, intervalo de confiança de 95\% percentis, valores mínimo e máximo e/ou de frequência absoluta e relativa, conforme a natureza da variável e apresentado em tabelas e ou gráficos. A análise foi feita a partir do coeficiente de correlação Pearson e Spearman e da análise Multivariada. Foram executados com o auxílio do programa BioEstat 5.5, sendo que resultados com $p \leq 0.05$ (bilateral) foram considerados estatisticamente significativos.

A coleta de dados foi realizada após aprovação do Comitê de Ética em Pesquisa com Seres Humanos do Centro Universitário do Estado do Pará, com
CAAE (09435319.7.0000.5169) e parecer de aprovação n. 3.234.958, aprovado em 31 de março de 2019.

\section{RESULTADOS}

Foram entrevistados 70 pacientes. 0 protocolo utilizado apresenta a caracterização sociodemográfica dos pacientes com Diabetes Mellitus usuários de insulina atendidos no CEMEC (Tabela 1), onde se observa que a maioria era do sexo feminino, com ensino fundamental completo ou incompleto, e renda de até um salário-mínimo.

A idade média dos pacientes foi igual a 55,4 anos (IC95\% 51,8 - 58,9), variando entre 15 e 88 anos. De acordo com as variáveis clínicas tempo médio de diagnóstico de DM foi igual a 42,9 anos (IC95\% 39,7 - 46,2), variando entre cinco e 78 anos.

Ao menos $50 \%$ dos pacientes tinham 46 anos ou menos ao ser diagnosticado. Em relação ao tempo médio de diagnóstico, este foi igual a 12,4 anos (IC95\% 10,3 - 14,6), variando entre um e 40 anos; a maioria teve seu diagnóstico há 20 anos ou menos $(p=0,0057)$.

A distribuição do tempo de insulinoterapia, entre os participantes 
foi igual a cinco anos (IC95\% 3,9 - 6,2), variando entre um e 25 anos, e ao menos $75 \%$ dos pacientes utilizavam insulina há seis anos ou menos tempo $(p<0.0001)$.
Quanto as aplicações diárias de insulina, observou-se que o número médio foi igual a 2,9 aplicações (IC95\% 2,6 - 3,2), entre um e seis vezes ao dia.

$\begin{array}{lccc}\text { Tabela } 1 \text { - Caracterização sociodemográfica de pacientes com } & \text { Diabetes } \\ \text { Mellitus usuários de insulina atendidos no Centro de Especialidades Médicas } \\ \text { do CESUPA. Fevereiro a julho de 2019. Belém (PA), Brasil. }\end{array}$

A distribuição dos escores do BPAID teve como resultado, a maioria $(64,3 \%)$ apresentava escore B-PAID elevado ( $\geq 40$ ), indicando alto nível de sofrimento emocional relacionado ao diabetes (Tabela 2).

Não se observou correlação estatística entre as subdimensões do BPAID com a idade, tempo de diagnóstico, o tempo de insulinoterapia e a frequência de aplicações.

No entanto, embora não significativa, nota-se correlação positiva entre o tempo de insulinoterapia e todas as subdimensões do B-PAID e o escore geral, ou seja, quanto maior o tempo de uso de insulina pior é QV do paciente. Em relação ao tempo de diagnóstico, a idade e a frequência de aplicações, é possivel notar as correlações entre estas variáveis e o escore B-PAID, indicando que quanto maior as variáveis, melhor a QV.

Não observou diferença estatisticamente significativa das subdimensões do B-PAID e escore geral em função do gênero (Tabela 3). Entretanto, foram observados valores 
medianos numericamente maiores na subdimensão alimentação entre as mulheres e nas subdimensões tratamento e apoio social entre os homens.

Além disso, o gênero feminino apresentou maiores médias nos escores do questionário B-PAID quando comparado com o masculino, indicando um maior impacto negativo na qualidade de vida das mulheres diabéticas.

Quanto à escolaridade, foram observados valores medianos numericamente menores no B-PAID geral e nas subdimensões emocional, alimentação e apoio social entre os pacientes com ensino superior, entretanto sem significância estatística. Com relação à renda foram encontrados valores medianos numericamente menores no B-PAID entre os pacientes com renda entre quatro e cinco salários mínimos (Tabela 4).

Tabela 2 - Comparação do B-PAID com as variáveis clínicas em pacientes com Diabetes Mellitus usuários de insulina. Fevereiro a julho de 2019. Belém (PA), Brasil.

\begin{tabular}{|c|c|c|c|}
\hline \multirow[b]{2}{*}{ Variável } & \multicolumn{2}{|c|}{ B-PAID } & \multirow[b]{2}{*}{$P$ valor } \\
\hline & $\begin{array}{l}<40 \\
\mathrm{n} ; \%\end{array}$ & $\begin{array}{c}\geq 40 \\
\mathrm{n} ; \%\end{array}$ & \\
\hline \multicolumn{4}{|l|}{ Idade } \\
\hline Média \pm desvio padrão & 55,9 & 55,1 & 0,8199 \\
\hline IC95\% & $49,2-62,6$ & $50,8-59,3$ & \\
\hline Mínimo - Máximo & $15,0-79,0$ & $25,0-88,0$ & \\
\hline \multicolumn{4}{|l|}{ Tempo de Diagnóstico } \\
\hline Mediana & $10,0(18,0)$ & $10,0(9,0)$ & \\
\hline Mínimo - Máximo & $1,0-40,0$ & $1,0-30,0$ & 0,3055 \\
\hline IC95\% & $8,0-20,0$ & $6,0-12,0$ & \\
\hline \multicolumn{4}{|c|}{ Tempo de insulinoterapia } \\
\hline Mediana & $2,0(2,0)$ & $4,0(4,0)$ & \\
\hline Mínimo - Máximo & $1,0-19,0$ & $1,0-25,0$ & 0,1003 \\
\hline IC95\% & $3,0-4,0$ & $2,0-3,0$ & \\
\hline \multicolumn{4}{|c|}{ Frequência de aplicações } \\
\hline Mediana & $3,0(2,0)$ & $3,0(2,0)$ & \\
\hline Mínimo - Máximo & $1,0-5,0$ & $1,0-6,0$ & 0,5871 \\
\hline IC95\% & $2,0-4,0$ & $3,0-5,0$ & \\
\hline
\end{tabular}


Tabela 3 - Distribuição dos pacientes com diabetes mellitus usuários de insulina. Fevereiro a julho de 2019. Belém (PA), Brasil.

\begin{tabular}{|c|c|c|c|c|}
\hline \multirow[t]{2}{*}{ Subdimensão B-PAID } & \multicolumn{3}{|c|}{ Renda (salários-mínimos) } & \multirow[t]{2}{*}{$P$ valor* } \\
\hline & $0|-| 1$ & $2|-| 3$ & $4|-| 5$ & \\
\hline \multicolumn{5}{|l|}{ Geral } \\
\hline Mediana (DIQ) & $50,0(33,1)$ & $55(38,7)$ & $41,9(55,3)$ & 0,6963 \\
\hline Mínimo - Máximo & $0-86,3$ & $15,0-82,5$ & $17,5-85,0$ & \\
\hline P25/P75 & $31,9 / 65,0$ & $33,8 / 72,5$ & $21,3 / 76,6$ & \\
\hline IC95\% & $38,8-58,8$ & $33,8-75,0$ & $27,5-40,0$ & \\
\hline \multicolumn{5}{|l|}{ Emocional } \\
\hline Mediana (DIQ) & $33,8(37,5)$ & $40,0(23,2)$ & $30,0(39,4)$ & 0,6774 \\
\hline Mínimo - Máximo & $0-58,8$ & $11,3-60,0$ & $15,0-57,5$ & \\
\hline P25/P75 & $21,3 / 58,8$ & $23,1 / 46,3$ & $18,1 / 57,5$ & \\
\hline IC95\% & $27,5-40,0$ & $20,0-47,5$ & $15,0-57,5$ & \\
\hline \multicolumn{5}{|l|}{ Alimentação } \\
\hline Mediana (DIQ) & $8,8(11,3)$ & $8,8(11,9)$ & $5,0(12,5)$ & 0,8330 \\
\hline Mínimo - Máximo & $0-15,0$ & $0-15,0$ & $0-15,0$ & \\
\hline P25/P75 & $2,5 / 13,8$ & $1,9 / 13,8$ & $0,6 / 13,1$ & \\
\hline IC95\% & $5,0-11,3$ & $0-15,0$ & $0-15,0$ & \\
\hline \multicolumn{5}{|l|}{ Tratamento } \\
\hline Mediana (DIQ) & $5,0(3,7)$ & $5,0(5,0)$ & $3,8(9,4)$ & 0,9174 \\
\hline Mínimo - Máximo & $0-15,0$ & $0-15,0$ & $0-10,0$ & \\
\hline P25/P75 & $3,8 / 7,5$ & $2,5 / 7,5$ & $0 / 9,4$ & \\
\hline IC95\% & $5,0-6,3$ & $0-7,5$ & $0-10,0$ & \\
\hline \multicolumn{5}{|l|}{ Apoio social } \\
\hline Mediana (DIQ) & $0(5,0)$ & $5,0(6,3)$ & $3,1(2,0)$ & 0,3240 \\
\hline Mínimo - Máximo & $0-10,0$ & $0-10,0$ & $0-5,0$ & \\
\hline P25/P75 & $0 / 5,0$ & $0 / 6,3$ & $0,3 / 5,0$ & \\
\hline IC95\% & $0-3,8$ & $0-7,5$ & $0-5,0$ & \\
\hline
\end{tabular}

Tabela 4 - Comparação do B-PAID com as variáveis sociodemográficas. Fevereiro a julho de 2019. Belém (PA), Brasil.

\begin{tabular}{|c|c|c|c|}
\hline \multirow[b]{2}{*}{ Variável } & \multicolumn{2}{|c|}{ B-PAID } & \multirow[b]{2}{*}{$P$ valor } \\
\hline & $\begin{array}{l}<40 \\
\mathrm{n} ; \%\end{array}$ & $\begin{array}{l}\geq 40 \\
\mathrm{n} ; \%\end{array}$ & \\
\hline \multicolumn{4}{|l|}{ Gênero } \\
\hline Masculino & $08 ; 32$ & $17 ; 37,8$ & \multirow[t]{2}{*}{0,7954} \\
\hline Feminino & $17 ; 68$ & $28 ; 62,2$ & \\
\hline \multicolumn{4}{|l|}{ Escolaridade } \\
\hline Analfabeto/apenas lê e escreve & $05 ; 20$ & $08 ; 17,8$ & \multirow{4}{*}{0,6116} \\
\hline Ensino fundamental (completo/incompleto) & $09 ; 36$ & $22 ; 48,9$ & \\
\hline Ensino médio (completo/incompleto) & $08 ; 32$ & $13 ; 28,9$ & \\
\hline Ensino superior (completo/incompleto) & $03 ; 12$ & $02 ; 4,4$ & \\
\hline \multicolumn{4}{|l|}{ Renda (salários mínimos) } \\
\hline $0-1$ & $19 ; 67,9$ & $34 ; 75,6$ & \multirow{3}{*}{0,1823} \\
\hline $2-3$ & $04 ; 14,3$ & $09 ; 20$ & \\
\hline $4-5$ & $05 ; 17,8$ & $02 ; 4,4$ & \\
\hline
\end{tabular}




\section{DISCUSSÃO}

O grupo estudado caracterizou por baixo nível socioeconômico, em sua maioria do gênero feminino e usuários do SUS. O nível de sofrimento emocional relacionado a diabetes demonstrou valores semelhantes a estudo anterior ${ }^{19}$.

Observou-se que os escores do BPAID entre os pacientes estudados nesta pesquisa apresentaram elevados $(\geq 40)$, indicando maior impacto na qualidade de vida. Em contrapartida, outro estudo $^{20}$ apontou que, de maneira geral, o DM não exerceu um aspecto negativo no emocional da população estudada. A diferença de resultados pode ser explicada pelo fato do diabetes trazer especificidades que variam de indivíduo para indivíduo e de uma população para outra, como sendo um fenômeno singular.

O presente estudo está de acordo com os dados apresentados em pesquisa realizada em Maringá (PR) com predomínio do sexo feminino. Esse dado pode estar associado a melhor percepção das doenças por este grupo e por buscarem mais pela assistência médica quando comparado ao gênero masculino ${ }^{21}$.

Pesquisa revela que o gênero feminino tem sido mais prevalente no cuidado com o diabetes em relação ao gênero masculino no quesito estilo de vida, controle alimentar, tempo de diagnóstico e realização de exames laboratoriais para o controle do diabetes. No entanto, o gênero masculino possui maior prevalência na prática de atividade física regular ${ }^{22}$.

Os dados do presente estudo revelaram que mesmo sem significância estatística, há um maior impacto na QV em pacientes de baixa renda. Os custos com o manejo ideal do diabetes, a auto monitoração e o uso de insulina que proporcionem maior comodidade $\mathrm{e}$ melhor controle glicêmico, dificultam o cuidado de grande parte dos diabéticos ${ }^{10}$.

Nesse sentido, os fatores socioeconômicos possuem uma relação direta com os hábitos de vida e a prática de autocuidado nos pacientes com DM. Por outro lado, esses fatores impõem aos profissionais da saúde um desafio maior no planejamento, abordagens e estratégias de cuidado para cada paciente ${ }^{23}$.

Em um estudo internacional, os pacientes com poucos anos de escolaridade, correlacionaram-se com um pior controle metabólico, pior percepção sob a QV e maiores sintomas depressivos $^{24}$. Equivalente a este, no 
artigo de validação do B-PAID observouse forte correlação negativa entre menores anos de escolaridade e 0 instrumento utilizado ${ }^{15}$.

Apesar de não evidenciar significância estatística, os resultados foram semelhantes a literatura ${ }^{21}$, ao demonstrar a maior tendência da amostra com a baixa escolaridade em apresentar escores baixos de QV. A escolaridade é um fator que pode limitar o acesso às informações tanto por comprometer a habilidade de leitura como pela compreensão adequadamente dos mecanismos da doença ${ }^{21}$.

Sobre o número de aplicações diárias de insulina, o presente estudo obteve valores semelhantes ao estudo realizado em Porto Alegre (RS) ${ }^{25}$ o qual demonstrou que a maioria dos pacientes com baixa QV faziam em torno de duas aplicações de insulina ao dia. Diversos fatores podem estar diretamente relacionados com a forma negativa de lidar com o uso de insulina, entre eles fatores sociais, psicológicos e efeitos adversos que atuam em conjunto na geração de medo e incertezas ${ }^{26}$.

Pesquisas que também utilizaram o questionário B-PAID, demonstraram maior impacto na QV entre os usuários de insulina, quando comparados aos pacientes diabéticos não usuários ${ }^{10,22,25}$.
Estudo em Santa Catarina (BR) sobre prevalência de transtornos de ansiedade em 996 pacientes insulinodependentes, concluiu que os participantes possuíam maior sofrimento psicológico, com comprometimento no desenvolvimento de atividades em geral ${ }^{27}$.

Pacientes em uso de insulina acumulam sentimento de culpa quando não conseguem obter níveis glicêmicos eficazes e, devido a isso, apresentam ideia pessimista sobre seu prognóstico. Esse fato gera transtornos para os usuários, o que poderia justificar um prejuízo maior na área referente aos problemas sociais ${ }^{28}$.

Em relação ao tempo de diagnóstico, este estudo mostrou uma tendência a piora da QV, quanto menor o tempo de descoberta do diabetes. Isso provavelmente ocorre, por terem menos tempo de adaptação com a doença e pela dificuldade em aceitar mudanças repentinas do estilo de vida, desde as aplicações diárias de insulina até restrições no comportamento alimentar $^{29}$.

Dessa forma, o maior tempo de diagnóstico repercute na maior compreensão do diabetes, assim como melhor manejo do esquema terapêutico. Portanto, intervenções na educação e nas habilidades específicas para 
enfrentar o diabetes podem melhorar a qualidade de vida dos pacientes com essa doença ${ }^{30}$.

Os homens apresentaram maior mediana na subdimensão tratamento e apoio social, enquanto nas mulheres a tendência foi maior a problemas com a alimentação. Esses resultados foram contrários ao estudo em que no gênero masculino o maior problema foi relacionado à alimentação e no feminino relacionado ao tratamento ${ }^{27}$. Tal diferença pode ser explicada pela variação sociocultural de cada população.

Pesquisa no Hospital da Universidade Federal de Sergipe (HUUFS) junto a dois grupos, um que recebia apenas atendimento pela equipe multiprofissional e outro que além do acompanhamento, participaram de grupo educativo (abordados diversos conteúdos desde a fisiopatologia do DM até cuidados com os pés e a importância da prática de atividades físicas), observou-se diminuição do sofrimento em viver com o DM somente com o grupo que integrou a atividade educativa ${ }^{31}$.

A promoção em saúde pode contribuir para diminuição dos índices glicêmicos ${ }^{32,33}$ e a modalidade de grupo é a forma mais potente para essa prática, por atingir um alvo maior de indivíduos, além de aproximá-los de seus familiares, profissionais de saúde e sua comunidade como um todo 5 . Entretanto, a equipe de saúde deve estar capacitada e motivada para esse acompanhamento e intervenção, a fim de detectar problemas e ajustar o tratamento, manejar intercorrências e valorizar/utilizar fatores emocionais em prol do autocuidado e controle da glicemia ${ }^{34}$.

Embora este estudo tenha apresentado limitações por não fazer comparativos com grupos não usuários de insulina, outros estudos corroboram para confirmar a redução na QV nesses pacientes quando comparados aos que realizam apenas terapêutica oral.

\section{CONCLUSÃO}

0 estudo permite concluir que dentre os pacientes entrevistados a maioria foi representada por idade avançada, com prevalência do sexo feminino, com ensino fundamental (incompleto/completo) e renda entre $0 \mathrm{e}$ 1 salários mínimos. A respeito do questionário B-PAID, prevaleceu o score elevado ( $\geq 40$ ), caracterizando como uma população com alto nível de sofrimento emocional relacionado ao diabetes. 
Diante disso, há necessidade de outros estudos para determinar se 0 resultado do sofrimento emocional dessas pessoas insulinodependentes tem relação com o próprio esquema de tratamento estabelecido em comparação com os demais perfis de diabéticos. Tais descobertas, podem auxiliar a avaliação criteriosa do grau de QV nesse grupo, lançando mão de todo suporte clínico para evitar o abandono do tratamento e as complicações das doenças.

\section{REFERÊNCIAS}

1. Ministério da saúde (BR). Protocolo clínico e diretrizes terapêuticas diabetes mellitus tipo 1. Brasília: CONITEC; 2019.

2. International Diabetes Federation [Internet]. IDF Diabetes Atlas - 8th Edition. Bruxelas: International Diabetes Federation; 1996. [acesso em 2018 dez. 19]. Disponível em: www.idf.org.br/diabetesatlas

3. Marinho NBP, Vasconcelos HCA, Alencar AMPG, Almeida PC, Damasceno MMC. Risco para diabetes mellitus tipo 2 e fatores associados. Acta Paul Enferm. 2013; 26(6):569574.

4. Oliveira JEP, Montenegro Júnior RM, Vencio S. Diretrizes da Sociedade
Brasileira de Diabetes 2017-2018. São Paulo: Clannad; 2017.

5. Andrade CJN, Alves CAD. Influence of socioeconomic and psychological factors in glycemic control in young children with tiype 1 diabetes mellitus. J Pediatr (Rio J). 2019; 95(1):48-53.

6. Lovre D, Fonseca V. Benefits of timely basal insulin control in patients with type 2 diabetes. J diabetes its complicat. 2015; 29(2):295-301.

7. Oliveira RA, Tostes $M$, Queiroz VA, Rodacki $M$, Zajdenverg L. Insulin mediated improvement in glycemic control in elderly with type 2 diabetes mellitus can improve depressive symptoms and does not seem to impair health-related quality of life. Diabetol metab syndr. 2015; 7:55.

8. Ishii $\mathrm{H}$, Terauchi $\mathrm{Y}$, Junnouci $\mathrm{H}$, Takeuchi M, Imaoka T. Effects of insulin changes on quality of life and glycemic control in Japanese patients with type 2 diabetes mellitus: the insulin-changing study intending to gain patients' insights into insulin treatment with patientreported health outcomes in actual clinical treatments (INSIGHTs) study. J diabetes Investig. 2013; 4(6):560- 
570.

9. Pichon-Riviere A, Irazola V, Beratarrechea A, Alcaraz A, Carrara C. Quality of life in type 2 diabetes mellitus patients requiring insulin treatment in Buenos Aires, Argentina: a cross-sectional study. Int $j$ health policy manag. 2015; 4(7):475-480.

10. Ramalho MRL, Marques TF, Silva JMFL, Silva GL. Qualidade de vida em pacientes diabéticos usuários de insulina na atenção secundária do Cariri cearense. Rev bras qual vida. 2017; 9(4):361-374.

11. Jackson IL, Adibe MO, Okonta MJ, Ukwe CV. Medication adherence in type 2 diabetes patients in Nigeria. Diabetes Technol Ther. 2015; 17(6):398-404.

12. Zioga E, Kazakos K, Dimopoulos E, Koutras C, Marmara K, Marmara EE, et al. Adherence and quality of life in patients with type II diabetes mellitus in northern greece. Mater Sociomed. 2016; 28(4):258-262.

13. Xin C, Xia Z, Jiang C, Lin M, Li G. Effect of pharmaceutical care on medication adherence of patients newly prescribed insulin therapy: a randomized controlled study. Patient Prefer Adherence. 2015; 9:797-802.

14. Gusmai LF, Novato TS, Nogueira LS.
The influence of quality of life in treatment adherence of diabetic patients: a systematic review. Rev Esc Enferm USP. 2015; 49(5):839.

15. Gross CC, Scain SF, Scheffel R, Gross $\mathrm{JL}$, Hutz CS. Brazilian version of the problems areas in diabetes scale (BPAID): validation and identification of individuals at high risk for emotional distress. Diabetes Res Clin Pract. 2007; 76(3):455-459.

16. Picanço LAA, Vieira EP, Lima AB, Byk J, Santos JOL. Evaluation instruments of the quality of life of individyals with diabetes mellitus. Arq Ciênc Saúde. 2017; 24(4):69-72.

17. Alpizar CMC, Valenciano LR. Intervenciones de enfermaría para mejorar la calidad de vida de las personas con pie diabético. J Health NPEPS. 2018; 3(2):566-582.

18. Kiosli K, Scott W, Winkley K, Kylakos S, McCracken LM. Psychosocial Factors in Painful Diabetic Neuropathy: A Systematic Review of Treatment Trials and Survey Studies. Pain med. 2019; 20(9):1756-1773.

19. Bernini LS, Barrile SR, Mangili AF, Arca EA, Correr R, Ximenes MA, et al. O impacto do diabetes mellitus na qualidade de vida de pacientes da Unidade Básica de Saúde. Cad Bras Ter Ocup. 2017; 25(25):533-541. 
20. Leite ES, Lubenow JAM, Moreira CRM, Martins MM, Costa PI, Silva AO. Avaliação do impacto da diabetes mellitus na qualidade de vida de idosos. Ciênc cuid saúde. 2015; 14(1):822-829.

21. Freitas SS, Silva GRF, Rezende Neta DS, Silvada ARV. Analysis of the selfcare of diabetics according to by the Summary of Diabetes Self-Care Activities Questionnaire (SDSCA). Acta Scientiarum Health Sci. 2014; 36(1):73-81.

22. Rossaneis MA, Haddad MCFL, Mathias TAF, Marcon SS. Differences in foot self-care and lifestyle between men and women with diabetes mellitus. Rev latinoam enferm. 2016; 24:e2761.

23. American Diabetes Association (ADA). Standards of medical care in diabetes-2015. Diabetes Care. 2015; 38(Supplement 1):S4-S4.

24. Papelbaum M, Lemos HM, Duchesne M, Kupfer R, Moreira RO, Coutinho WF. The association between quality of life, depressive symptoms and glycemic control in a group of type 2 diabetes patients. Diabetes Res Clin Pract. 2010; 89(3):227-30.

25. Soares JPG, Dell'aglio DD. Relaçones entre qualidade de vida e diabetes mellitus tipo 1 na adolescencia.
Contextos Clinn. 2016; 9(2):159-167.

26. Luk A. Psychological insulin resistance: scope of the problem. Hong Kong Med J. 2016; 22(4):304305.

27. Santos MAB, Ceretta LB, Réus GZ, Helena MA, Jornada LK, Schwalm MT, et al. Anxiety disorders are associated with quality of life impairment in patients with insulindependent type 2 diabetes: a casecontrol study. Rev bras psiquiatr. 2014; 36(4):298-304.

28. Abu HH, Tohid H, Mohd AR, Long Bidin MB, Muthupalaniappen L, Omar $\mathrm{K}$. Factors influencing insulin acceptance among type 2 diabetes mellitus patients in a primary care clinic: a qualitative exploration. BMC Family Practice. 2013; 164(4).

29. Gouvêa GR, Barbosa LFN, Silva MAV, Guerra LM, Pereira AC, Possobon RF, et al. Qualidade de vida e características dos pacientes diabéticos. Ciênc saúde colet. 2017; 22(3):921-930.

30. Faria HTG, Rodrigues FF, Zanetti ML, Araújo MF, Damasceno MM. Fatores associados à adesão ao tratamento de pacientes com diabetes mellitus. Acta Paul Enferm. 2013; 26(3):231237.

31. Brito GM, Gois CF, Zanetti ML, 
Resende GG, Silva JR. Qualidade de vida, conhecimento e atitude após programa educativo para Diabetes. Acta Paul Enferm. 2016; 29(3):298306.

32. Grillo MF, Neumann CR, Scain SF, Rozeno RF, Gross JL, Leitão CB. Efeito de diferentes modalidades de educação para o autocuidado a pacientes com diabetes. Rev Assoc Med Bras. 2013; 59(4):400-405.

33. Herrera VR, Zéron HM, Alcántara MR.
Adherence to two methods of education and metabolic control in type 2 diabetics. Ethiop J Health. 2015; 25(2):163-70.

34. Santamaria YR, Cabriales ECG, Valverde JMG, Luna NSA. Teoria de auto-manejo y control glucémico em adultos com diabetes mellitus tipo 2 . J Health NPEPS. 2017; 2(2):444-456.

Financiamento: Os autores declaram que não houve financiamento.

Conflito de interesses: Os autores declaram não haver conflito de interesses.

\section{Participação dos autores:}

- Concepção: Aviz GB, Santos FM, Azevedo VDC, Silva GG, Furtado LL.

- Desenvolvimento: Aviz GB, Santos FM, Azevedo VDC, Silva GG, Furtado LL.

- Redação e revisão: Aviz GB, Santos FM, Azevedo VDC, Silva GG, Furtado LL.

Como citar este artigo: Aviz GB, Santos FM, Azevedo VDC, Silva GG, Furtado LL. Avaliação da qualidade de vida e perfil socioeconômico em diabéticos insulinodependentes. J Health NPEPS. 2021; 6(1):47-61.

Submissão: 20/06/2020

Aceito: 25/09/2020

Publicado: 01/06/2021 